\title{
CORONAGRAPHIC OBSERVATIONS OF FOMALHAUT AT SOLAR SYSTEM SCALES
}

\author{
Matthew A. Kenworthy ${ }^{1}$, Tiffany Meshiat ${ }^{1}$, Sascha P. Quanz ${ }^{2}$, Julien H. Girard ${ }^{3}$, \\ Michael R. Meyer ${ }^{2}$, AND Markus KaSPeR ${ }^{4}$ \\ ${ }^{1}$ Leiden Observatory, Leiden University, P.O. Box 9513, 2300 RA Leiden, The Netherlands \\ 2 Institute for Astronomy, ETH Zurich, Wolfgang-Pauli-Strasse 27, CH-8093 Zurich, Switzerland \\ ${ }^{3}$ European Southern Observatory, Alonso de Cordova 3107, Vitacura, Cassilla 19001, Santiago, Chile \\ ${ }^{4}$ European Southern Observatory, Karl Schwarzschild Strasse, 2, D-85748 Garching bei Munchen, Germany \\ Received 2012 September 21; accepted 2012 December 5; published 2013 January 18
}

\begin{abstract}
We report on a search for low mass companions within $10 \mathrm{AU}$ of the star Fomalhaut, using narrowband observations at $4.05 \mu \mathrm{m}$ obtained with the Apodizing Phase Plate coronagraph on the VLT/NaCo. Our observations place a model-dependent upper mass limit of $12-20 M_{\text {jup }}$ from 4 to $10 \mathrm{AU}$, covering the semimajor axis search space between interferometric imaging measurements and other direct imaging non-detections. These observations rule out models where the large semimajor axis for the putative candidate companion Fomalhaut $\mathrm{b}$ is explained by dynamical scattering from a more massive companion in the inner stellar system, where such giant planets are thought to form.
\end{abstract}

Key words: instrumentation: high angular resolution - planetary systems - planets and satellites: detection stars: individual (Fomalhaut) - techniques: high angular resolution

Online-only material: color figure

\section{INTRODUCTION}

The formation and distribution of the planets in our solar system is closely entwined with the early evolution of its debris disk (Lissauer 1993) which, despite many different lines of evidence, do not produce a clear picture. By studying debris disks with a range of stellar ages, however, an evolutionary picture can be formed (see the reviews of Wyatt et al. 2007; Meyer et al. 2007). The morphology of dust in debris disks around nearby stars is thought to be a signpost of planet formation, leading to an intense study of the nearest debris disk systems to look for the bodies that are sculpting these structures. Deep imaging surveys therefore have focused on the closest systems where spatial resolution provides the greatest detail. Fomalhaut is a nearby $(d=7.7 \mathrm{pc}) \mathrm{A} 3 \mathrm{~V}$ star with an estimated age of $440 \pm 40 \mathrm{Myr}$ (Mamajek 2012). It has an inclined eccentric debris disk first resolved by Holland et al. (1998) in the sub-mm and subsequently imaged by the Hubble Space Telescope by Kalas et al. (2005). The sharp inner edge of the resolved ring and its eccentricity implies the gravitational presence of a planet (Quillen 2006) for which a candidate was imaged by Kalas et al. (2008) at optical wavelengths.

Observations taken with the Herschel Space Observatory from $70 \mu \mathrm{m}$ to $500 \mu \mathrm{m}$ (Acke et al. 2012) show that there is an estimated 110 Earth mass cometary reservoir supplying the dusty grains that are directly detected at these wavelengths. High angular resolution observations at $350 \mathrm{GHz}$ taken with ALMA resolve the debris ring on one side of Fomalhaut (Boley et al. 2012). The structure of the ring implies that the outer edge of the parent body ring is consistent with being as sharply truncated as the inner edge. The most likely explanation is the presence of unseen shepherding planetary bodies on either side of the ring.

We report on the search for a substellar companion to Fomalhaut down to $3 \mathrm{AU}$ of the star. We are motivated by the presence and location of the object labeled Fomalhaut b, which resides approximately $120 \mathrm{AU}$ in projection at the inner edge of the debris ring. The nature of Fomalhaut $b$ is uncertain.
The initial detection of reflected light in Kalas et al. (2008) was made at visible wavelengths, but subsequent observations do not detect the expected thermal emission from a massive gas giant (Marengo et al. 2009; Janson et al. 2012). The continuing existence of the dust ring implies a mass of less than three Jupiter masses, a prediction that is confirmed by a Spitzer nondetection of Fomalhaut $\mathrm{b}$ which limits its mass to $1 M_{\text {jup }}$ (Janson et al. 2012). The distance of Fomalhaut $b$ from its parent star means that the reflected light detection from the atmospheric surface of a Jupiter mass planet is not possible. Instead, the blue colors of the object imply that we are seeing starlight scattered from a cloud of dust which is either organized in a ring system about an unseen central object or a recently formed cloud of ejecta from a collision of planetesimals. A third alternative is that we are seeing a resonant clump of dust formed by unseen perturbation in the debris ring system.

It is a challenge to current planet formation theories to explain the presence of a massive object in an orbit at that distance from its central star, leading to the hypothesis that such planets are captured from another star during the early stages of stellar cluster evolution (Parker \& Quanz 2012), or that the object formed much closer to the parent star and was then dynamically ejected out to $120 \mathrm{AU}$ through gravitational interactions with one or more massive planets in the inner Fomalhaut system (Chiang et al. 2009). Additionally, it is interesting in its own right to look for giant planets around early-type stars, given that the first gas giant exoplanet detections have been around the early-type stars $\beta$ Pictoris (Lagrange et al. 2009, 2010; Quanz et al. 2010) and HR 8799 (Marois et al. 2008, 2010; Skemer et al. 2012), and to this end direct imaging surveys are producing statistically significant samples for analysis and interpretation (Ehrenreich et al. 2010; Janson et al. 2011; Vigan et al. 2012).

In Section 2 we describe the observations and data reduction carried out with $\mathrm{NaCo}$ at the Very Large Telescope (VLT), in Section 3 we carry out an analysis and interpretation of the presented data in terms of limits to faint companions of Fomalhaut. In Section 4, we compare our search with 
Table 1

Summary of Observations of Fomalhaut in Pupil Tracking Mode

\begin{tabular}{lccc}
\hline \hline Parameter & Hemisphere 1 & Hemisphere 2 & Hemisphere 3 \\
\hline UT date & 2011 Jul 19 & 2011 Aug 9 & 2011 Aug 7 \\
UT start & $07: 27: 43$ & $06: 08: 23$ & $06: 04: 17$ \\
UT end & $08: 42: 30$ & $07: 25: 20$ & $07: 15: 04$ \\
NDIT × DIT & $200 \times 0.23 \mathrm{~s}$ & $200 \times 0.23 \mathrm{~s}$ & $200 \times 0.23 \mathrm{~s}$ \\
Number of cubes & 75 & 78 & 72 \\
Parallactic angle start & -50.2 & $-46: 1$ & -58.9 \\
Parallactic angle end & 69.3 & $71: 7$ & 61.8 \\
Airmass & $1.005-1.023$ & $1.005-1.014$ & $1.005-1.028$ \\
Typical DIMM seeing & $0.60-0.75$ & $0.61-0.70$ & $0.83-1.32$ \\
\hline
\end{tabular}

other companion searches around Fomalhaut and how future observations with the Apodizing Phase Plate (APP) will be improved, and in Section 5 we present our conclusions.

\section{OBSERVATIONS AND DATA REDUCTION}

Data were obtained using $\mathrm{NaCo}$ (Lenzen et al. 2003; Rousset et al. 2003) on UT4 at Paranal in 2011 July and August (see Table 1). The conditions were photometric with no cloud cover. $\mathrm{NaCo}$ is run with the target, Fomalhaut, used as a natural guide star and the visible band wavefront sensor.

$\mathrm{NaCo}$ is used with the L27 camera, the NB4.05 filter $\left(\lambda_{c}=\right.$ $4.051 \mu \mathrm{m}$ and $\Delta \lambda=0.02 \mu \mathrm{m})$ and the APP Coronagraph used to provide additional diffraction suppression (Kenworthy et al. 2010; Quanz et al. 2010). Changes in the optical figure of the telescope mirrors and science camera optics lead to changes in the intensity of the telescope point spread function (PSF) at the science camera detector. The pupil tracking mode is therefore used to minimize the number of optical surfaces rotating with respect to the orientation of the detector, removing an additional component of PSF variation. The plane of the sky therefore rotates with respect to the camera focal plane.

Data are obtained in data cubes consisting of 200 sequential images, each one with a duration of $0.23 \mathrm{~s}$. One or more data cubes are obtained in one pointing, and then the telescope is moved by 6 arcsec along the top edge of the detector and another data cube acquired. For these observations we use a three point dither pattern along the top third of the $\mathrm{NaCo}$ detector. The APP coronagraph provides diffraction suppression over a $180^{\circ}$ wedge on one side of the star, at a cost of brighter diffraction (and increased noise) on the other side of the stellar PSF, resulting in a "dark side" and "bright side." In all subsequent data reduction, the bright side of the stellar PSF is masked off and removed from further analysis. Observations in pupil tracking mode result in a rotation of the "dark side" of the PSF on the sky. In order to obtain high contrast sensitivity for all position angles (PAs) around the target star, at least one additional observing block is required with the NaCo rotation set so that they directly complement the first set of observations in PA. In the case for Fomalhaut we have three separate sets of APP observations taken on separate nights (with PA offsets of $0^{\circ}, 120^{\circ}$, and $240^{\circ}$ ) that are combined to form a final image with $360^{\circ}$ of sensitivity. We deliberately saturate the PSF core so that the sensitivity to faint companions is increased. These observations are listed in Table 1. There are two sources of loss of sensitivity compared to direct imaging-first, the requirement to have two complementary PAs to provide the angular coverage, and a second loss in the core flux of all point sources in the image when using the APP. The APP coronagraph uses $45 \%-50 \%$ of the core flux to provide suppression of the

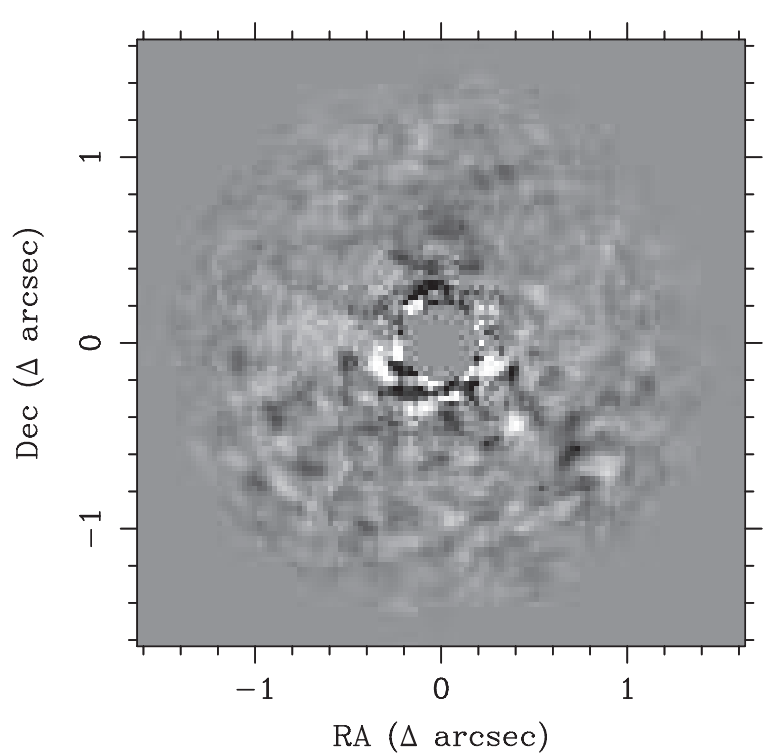

Figure 1. Image of Fomalhaut with fake companion added at $\mathrm{PA}=225$ and separation of $0.6 \operatorname{arcsec}(4.6 \mathrm{AU})$. The companion is 11 mag fainter than Fomalhaut and is detected with a signal to noise of 8.7 .

diffraction rings, and this leads to an increase of the integration time to reach a desired background limited signal-to-noise $(\mathrm{S} / \mathrm{N})$ limit. However, coronagraphs are always used at small angular separations where the dominant noise source is due to diffraction structures, and so there is a net gain in sensitivity when using a coronagraph in these conditions.

For the photometric calibration we used unsaturated images of Fomalhaut, itself a bright photometric standard star (van der Bliek et al. 1996) for the near-IR. Based on the unpublished UKIRT bright standard star catalog (see Leggett et al. 2003 for a discussion) the UKIRT $L^{\prime}$ magnitude of Fomalhaut is $0.96 \pm 0.01$. Since $\left(K-L^{\prime}\right)=0 \pm 0.02$ for Fomalhaut and the UKIRT $L^{\prime}$ filter is narrower than that of the more recent MKO-NIR filter set, we take the photometry of Fomalhaut in the NB4.05 filter to be $0.96 \pm 0.02$. The observing strategy and dither positions for Hemisphere 1 were used with the detector integration time (DIT) set to $0.0203 \mathrm{~s}$, and six data cubes were obtained. We follow the general data reduction approach in Quanz et al. (2010). With the large amount of sky rotation in the images, we use Angular Differential Imaging (Marois et al. 2006) in combination with the LOCI algorithm (Lafrenière et al. 2007) to subtract the stellar PSF of our images. For each science image, LOCI constructs a linear combination of the other science images that have enough sky rotation to avoid faint companion self-subtraction. The coefficients for the linear combination are calculated for different radii from the central star and for parameters describing the optimization region.

\section{RESULTS AND ANALYSIS}

\subsection{Point-source Detection Limits}

In Figure 1 we show the final PSF-subtracted image for Fomalhaut, together with an artificially inserted point source 11 mag fainter than Fomalhaut at $\mathrm{PA}=225^{\circ}$.

Fake planets are added to the raw data cubes to determine the limiting magnitude achievable. The unsaturated PSF image of Fomalhaut is used to create the fake planets in the saturated science images. They are added at angular separations incrementing from 0.2 to $1^{\prime \prime}$ in steps of $0^{\prime} ! 1$, and delta magnitudes 


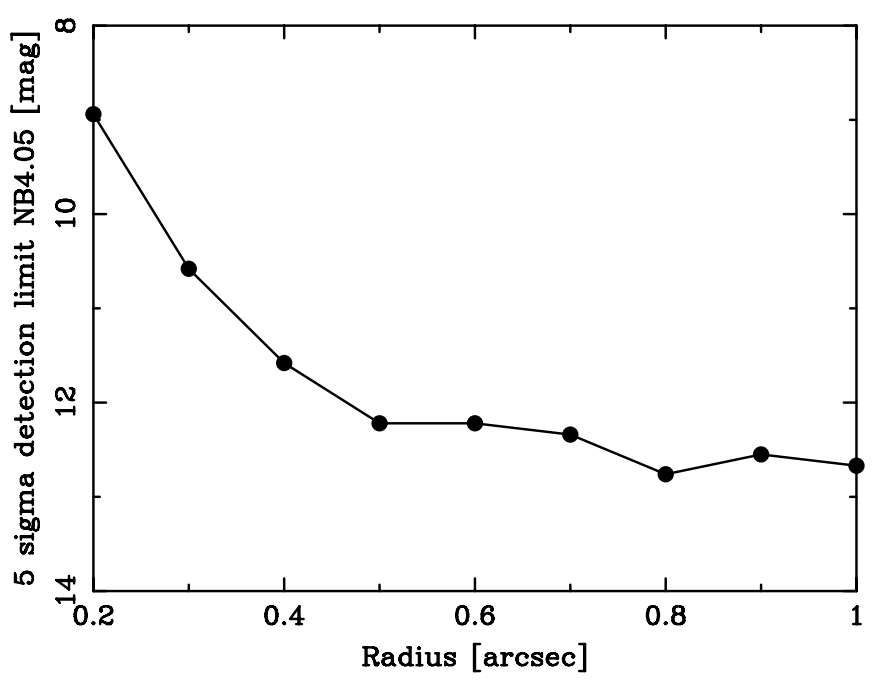

Figure 2. Sensitivity curve derived from artificially inserted and extracted point sources around Fomalhaut, with the sensitivity expressed in apparent NB4.05 magnitudes for a $5 \sigma$ point-source detection and angular separation in arcseconds. To convert to the contrast curve, subtract 0.96 mag (see the text for discussion).

in steps of $1 \mathrm{mag}$ from 8 to 14. The LOCI algorithm is used to extract the artificial planet while minimizing the speckles. We performed Monte Carlo simulations to determine the optimal parameters for the LOCI algorithm that yield the highest $\mathrm{S} / \mathrm{N}$ for the planet. The $\mathrm{S} / \mathrm{N}$ for the planet is defined as follows:

$$
\left(\frac{\mathrm{S}}{\mathrm{N}}\right)_{\text {planet }}=\frac{F_{\text {planet }}}{\sigma(r) \sqrt{\pi r_{\mathrm{ap}}^{2}}},
$$

where $F_{\text {planet }}$ is the sum of the planet flux in an aperture with radius $r_{\mathrm{ap}}=3$ pixels and $\sigma$ is the root mean square of the pixels in a $170^{\circ}$ wedge at the same radius, surrounding the star. After running the three hemispheres through LOCI, the output was collapsed into one final image with the best planet $\mathrm{S} / \mathrm{N}$. We extrapolate between the planet magnitudes to determine the magnitude that yields an $\mathrm{S} / \mathrm{N}$ of 5 . This process was repeated for all angular separations. Fake planets were added at four different PAs around Fomalhaut. The limiting magnitudes at each PA were calculated and the resultant mean limiting magnitude is shown in Figure 2.

To convert a given point source's absolute magnitude to the mass of a companion, we require both its age and a theory for the luminous evolution of the object. We convert the contrast curve into an upper limit of mass using the gas giant atmospheric models of Baraffe et al. (2003) and Spiegel \& Burrows (2012) to form the resultant contrast curve in Figure 3. The Spiegel \& Burrows (2012) models are solar metallicity with hybrid clouds. The blue curve is extrapolated from Figure 7 in Spiegel $\&$ Burrows (2012) to include masses greater than $10 M_{J}$ in the NB4.05 filter. The hot- and cold-start models are nearly equivalent for objects older than $400 \mathrm{Myr}$, hence only one curve is shown. No significant point sources are identified above the local azimuthally averaged background out to 1.5 arcsec (11.6 AU) around Fomalhaut. Our sensitivity in mass is flat from 0.6 to $1.5 \operatorname{arcsec}$ (4.6 to 11.6 AU), with an upper mass limit of $16 M_{\text {jup }}$ (Spiegel \& Burrows 2012) to $12 M_{\text {jup }}$ (Baraffe et al. 2003).

Our sensitivity to planetary mass objects is limited by the contrast achievable using the APP optic and by the recently revised age of the Fomalhaut system (Mamajek 2012). The

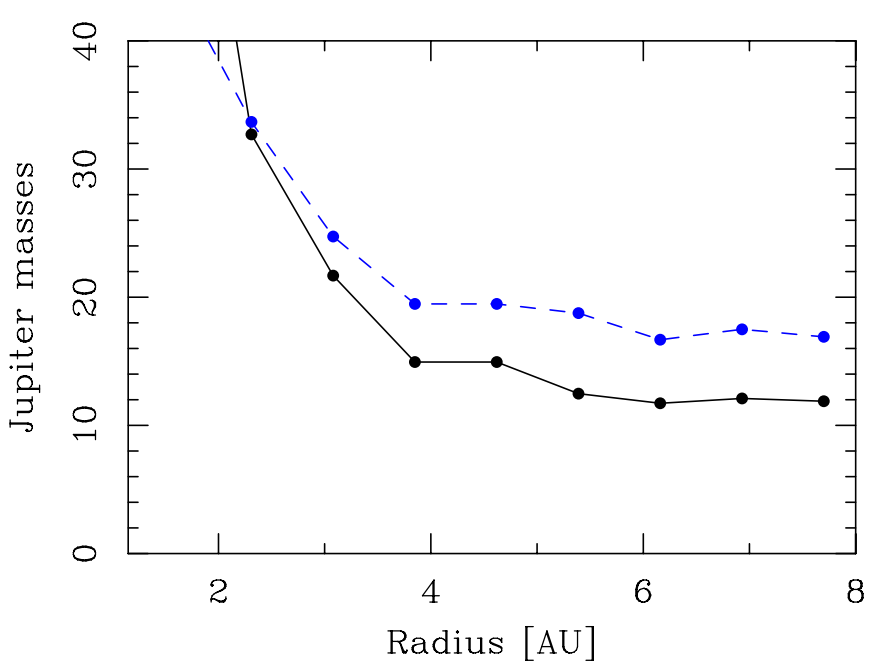

Figure 3. Detection limits for gas giant companions around Fomalhaut using Baraffe et al. (2003; solid black line) and Spiegel \& Burrows (2012; dashed blue line) models and assuming a distance of $7.81 \mathrm{pc}$ for Fomalhaut with the best LOCI parameter case.

(A color version of this figure is available in the online journal.)

luminosity of giant planets decreases as a function of time, and previous detection limits have taken an age of Fomalhaut of 250 Myr (Barrado y Navascues et al. 1997), based on several different aging techniques on both Fomalhaut and its associated companion TW PsA. In Mamajek (2012) the companionship is further confirmed and an improved isochronal age increases the age to $440 \pm 40 \mathrm{Myr}$, which is the age we adopt in this paper. The consequence of the increase in age means that previous mass sensitivities are approximately doubled, and our results are limited down to the high end of the planetary mass regime.

\section{DISCUSSION}

Several groups have searched for massive companions in the Fomalhaut system and these are shown in Figure 4, both as a point-source contrast plot and an upper mass limit plot using the giant planet models of Baraffe et al. (2003) and Spiegel \& Burrows (2012). The VLTI/PIONIER interferometer using the auxiliary $1.8 \mathrm{~m}$ telescopes at the $H$ band gives the largest angular separation coverage in logarithmic space from 1 mas to 100 mas with a peak sensitivity of $3 \times 10^{-3}$, scaled for a point-source contrast limit at $5 \sigma$ (Absil et al. 2011). This angular separation regime is not accessible to single dish telescopes, and future observations with the full $8.4 \mathrm{~m}$ telescopes in the VLTI will be able to look within $1 \mathrm{AU}$ of Fomalhaut and other nearby bright stars.

This work searches from 0.2 to 1.2 arcsec using a coronagraphic optic that provides additional diffraction suppression and provides an additional 1-1.5 mag of sensitivity compared to direct imaging sensitivities obtained with the same wavelength and instrument (Quanz et al. 2012). These observations overlap with the thermal infrared $(4.7 \mu \mathrm{m})$ measurements from Kenworthy et al. (2009) to look for companions out to $35 \mathrm{AU}$, where the planet detection limit reaches down to $2.6 \mathrm{M}_{\text {jup }}$ (using the models of Baraffe et al. 2003 and the revised age of $440 \mathrm{Myr}$ ), taking advantage of the thermal imaging performance of the $6.5 \mathrm{~m}$ MMTO telescope (Lloyd-Hart 2000) with the Clio thermal infrared imager (Hinz et al. 2006) and deformable secondary AO mirror system (Wildi et al. 2002; Brusa et al. 2003a). The observations in Kenworthy et al. (2009) are complete 


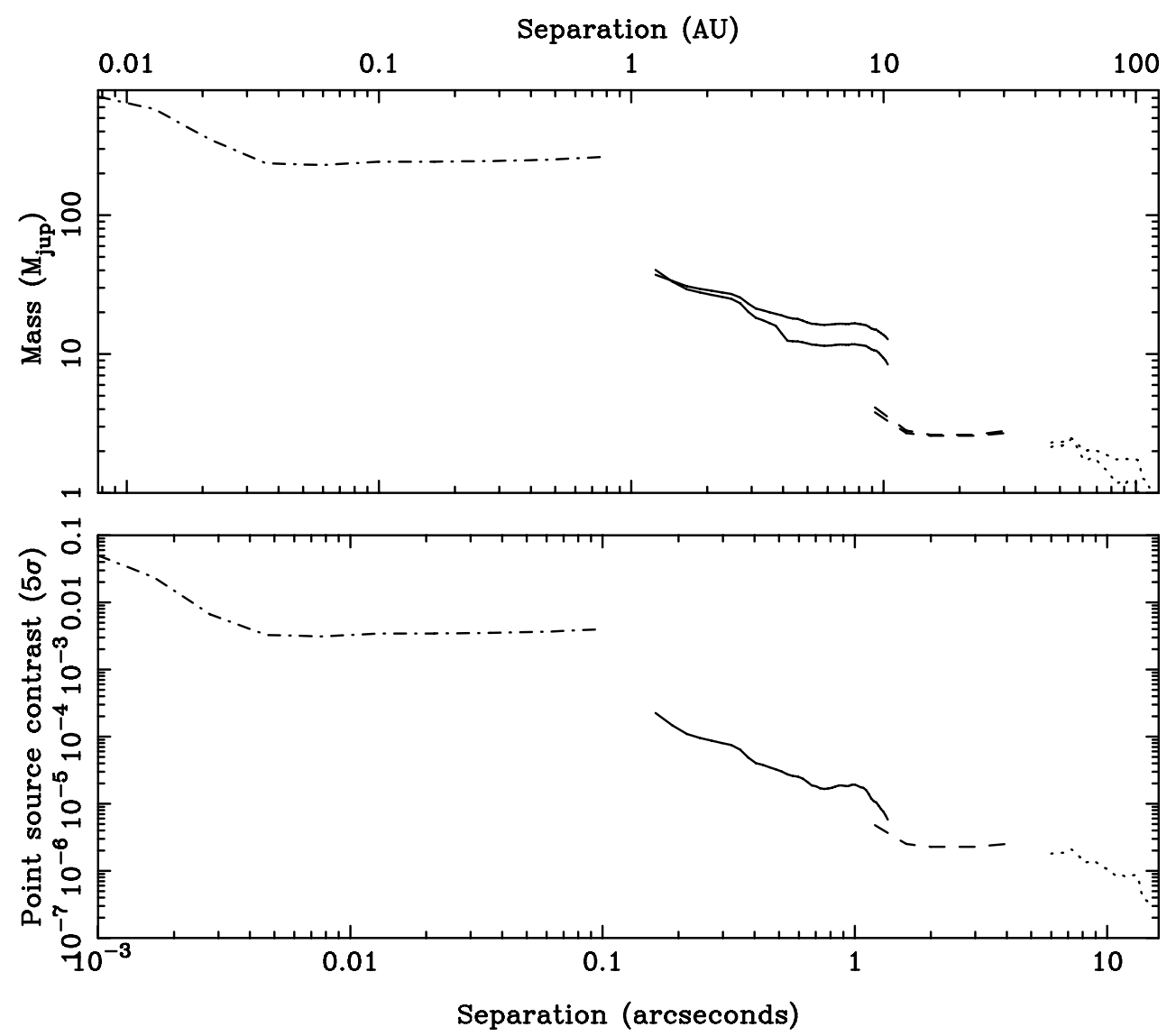

Figure 4. Contrast curves and upper mass limits for point sources around Fomalhaut compiled with measurements from the literature. From small to large angular separations, the curves represent data from Absil et al. (2011) [dot-dashed line-VLTI/1.8 m/1.65 $\mu \mathrm{m}$ ], this paper [solid line-VLT/8.4 m/4.05 $\mu \mathrm{m}$ ], Kenworthy et al. (2009) [dashed line-MMT/6.35 m/4.7 $\mu \mathrm{m}$ ], and Janson et al. (2012) [dotted line—Spitzer $/ 0.85 \mathrm{~m} / 4.5 \mu \mathrm{m}$ ]. All curves have been adjusted to $5 \sigma$ point-source sensitivity. Masses for the VLTI observations were estimated using the models of Baraffe et al. (1998) and the others from the models of Baraffe et al. (2003) and (Spiegel \& Burrows 2012). The age of Fomalhaut is taken to be 440 Myr (Mamajek 2012).

out to 4.5 arcsec, and additionally provide partial coverage to 10 arcsec. We restrict the contrast curve in Figure 4 to the complete coverage.

Others have looked for the thermal signature of Fomalhaut $\mathrm{b}$ using the Spitzer Space Telescope (Marengo et al. 2009; Janson et al. 2012) with model-dependent upper mass limits of $1 M_{\text {jup }}$ (Janson et al. 2012) being reached with the lower background sensitivity achievable in space. This also provides a constraint on companions in to 11 arcsec.

The contrasts attained at 1.3 arcsec between the MMT and VLT observations are comparable while there is a factor of two in planet mass sensitivity achieved. This is due to the extremely red colors predicted (and seen) in extrasolar giant planet models for younger systems $(<500 \mathrm{Myr})$, demonstrating the utility of carrying out extrasolar planet searches at thermal wavelengths around nearby stars (Hinz et al. 2006; Heinze et al. 2010) with both current facilities (LMIRCam Wilson et al. 2008) and future ELTs such as the E-ELT (METIS; Brandl et al. 2012) and GMT (TIGER; Hinz et al. 2012). By optimizing the telescope for thermal infrared observations with a deformable secondary mirror (Brusa et al. 2003b), fainter planets can be detected (Lloyd-Hart 2000).

There is a gap in coverage from 100 mas to 200 mas, which can be bridged with Sparse Aperture Masking (SAM) techniques (Nakajima et al. 1989; Tuthill et al. 2006, 2000) to cover 100-500 mas. This technique is well suited to bright targets such as Fomalhaut, and it is expected that this semimajor axis region will be observed in due course.
As can be seen, there is a large degree of coverage in semimajor axis around Fomalhaut, ruling out the presence of massive planetary mass companions to within approximately $3 \mathrm{AU}$ and brown dwarf companions within 2 AU. Further epochs will also close out the phase space that remains open due to the non-simultaneity of the reported observations.

Crida et al. (2009) suggest that a pair of massive planets at 5-20 AU could undergo a change in their semimajor axes through a mean motion resonance, with the larger companion remaining at $75 \mathrm{AU}$ while the outer one moves out to form the core for Fomalhaut $b$. The search presented here, in combination with other ground- and space-based observations, rules out the presence of brown dwarf mass companions. The need for a large, inner system scattering planet, however, may not be required to form Fomalhaut $b$ out at the large distance from the central star that it currently appears. Lambrechts \& Johansen (2012) suggest that gas giant cores can be rapidly accreted at large stellar distances in the early gas-rich phase of the protoplanetary disk by pebble accretion, obviating the need for gravitational scattering from the inner stellar system.

\section{SUMMARY AND CONCLUSIONS}

We have presented upper limits to massive companions in the Fomalhaut system, covering the range of semimajor axes from 1.5 AU to $11 \mathrm{AU}$. We detect no companions greater than a model dependent $20 M_{\text {jup }}$ from $4 \mathrm{AU}$ to $10 \mathrm{AU}$, and combining this with other searches in the literature we can rule any companion 
greater than $30 M_{\text {jup }}$ from 2.5 AU outward. The Fomalhaut system is older than previously thought, leading to an upward revision of the previously determined masses. We also rule out any brown dwarf that can cause the anomalous astrometric motion of Fomalhaut in Hipparcos data as suggested by Chiang et al. (2009). In the case of Fomalhaut, SAM observations are expected to close the gap for $0.1-0.5$ arcsec.

Future research is needed to confirm that the contrast limits at small angular separations are now dominated by non-common path errors between the wavefront sensor optical path and the science camera optical path. Efforts to measure and remove these errors have been demonstrated on $\mathrm{NaCo}$ with extra-focal imaging (Riaud et al. 2012) and by using the residuals from the wavefront sensor camera in a closed loop AO system (Codona et al. 2008).

This research has made use of the SIMBAD database, operated at CDS, Strasbourg, France. We are indebted to the ESO Paranal Support staff at the VLT, and to the anonymous referee for their comments on this paper. The data here were obtained under ESO Observing Run Number 087.C-0701(B).

Facility: VLT:Yepun

\section{REFERENCES}

Absil, O., Le Bouquin, J.-B., Berger, J.-P., et al. 2011, A\&A, 535, A68 Acke, B., Min, M., Dominik, C., et al. 2012, A\&A, 540, A125 Baraffe, I., Chabrier, G., Allard, F., \& Hauschildt, P. H. 1998, A\&A, 337, 403 Baraffe, I., Chabrier, G., Barman, T. S., Allard, F., \& Hauschildt, P. H. 2003, A\&A, 402, 701

Barrado y Navascues, D., Stauffer, J. R., Hartmann, L., \& Balachandran, S. C. 1997, ApJ, 475, 313

Boley, A. C., Payne, M. J., Corder, S., et al. 2012, ApJL, 750, 21

Brandl, B. R., Lenzen, R., Pantin, E., et al. 2012, Proc. SPIE, 8446, 84460A

Brusa, G., Riccardi, A., Salinari, P., et al. 2003a, Proc. SPIE, 4839, 691

Brusa, G., Riccardi, A., Wildi, F. P., et al. 2003b, Proc. SPIE, 5169, 26

Chiang, E., Kite, E., Kalas, P., Graham, J. R., \& Clampin, M. 2009, ApJ, 693, 734

Codona, J. L., Kenworthy, M. A., \& Lloyd-Hart, M. 2008, Proc. SPIE, $7015,70155 \mathrm{D}$

Crida, A., Masset, F., \& Morbidelli, A. 2009, ApJL, 705, 148

Ehrenreich, D., Lagrange, A., Montagnier, G., et al. 2010, A\&A, 523, A73
Heinze, A. N., Hinz, P. M., Sivanandam, S., et al. 2010, ApJ, 714, 155

Hinz, P., Codona, J., Guyon, O., et al. 2012, Proc. SPIE, 8446, 84460N

Hinz, P. M., Heinze, A. N., Sivanandam, S., et al. 2006, ApJ, 653, 1486

Holland, W. S., Greaves, J. S., Zuckerman, B., et al. 1998, Natur, 392,788

Janson, M., Bonavita, M., Klahr, H., et al. 2011, ApJ, 736, 89

Janson, M., Carson, J. C., Lafrenière, D., et al. 2012, ApJ, 747, 116

Kalas, P., Graham, J. R., Chiang, E., et al. 2008, Sci, 322, 1

Kalas, P., Graham, J. R., \& Clampin, M. 2005, Natur, 435, 1067

Kenworthy, M. A., Mamajek, E. E., Hinz, P. M., et al. 2009, ApJ, 697, 1928

Kenworthy, M., Quanz, S., Meyer, M., et al. 2010, Msngr, 141, 2

Lafrenière, D., Marois, C., Doyon, R., Nadeau, D., \& Artigau, É. 2007, ApJ, 660,770

Lagrange, A.-M., Bonnefoy, M., Chauvin, G., et al. 2010, Sci, 329, 57

Lagrange, A.-M., Gratadour, D., Chauvin, G., et al. 2009, A\&A, 493, L21

Lambrechts, M., \& Johansen, A. 2012, A\&A, 544, A32

Leggett, S. K., Hawarden, T. G., Currie, M. J., et al. 2003, MNRAS, 345, 144

Lenzen, R., Hartung, M., Brandner, W., et al. 2003, Proc. SPIE, 4841, 944

Lissauer, J. J. 1993, ARA\&A, 31, 129

Lloyd-Hart, M. 2000, PASP, 112, 264

Mamajek, E. E. 2012, ApJL, 754, 20

Marengo, M., Stapelfeldt, K., Werner, M. W., et al. 2009, ApJ, 700, 1647

Marois, C., Lafrenière, D., Doyon, R., Macintosh, B., \& Nadeau, D. 2006, ApJ, 641, 556

Marois, C., Macintosh, B., Barman, T., et al. 2008, Sci, 322, 1348

Marois, C., Macintosh, B., \& Véran, J. 2010, Proc. SPIE, 7736, 77361J

Meyer, M. R., Backman, D. E., Weinberger, A. J., \& Wyatt, M. C. 2007, in Protostars and Planets V, ed. B. Reipurth, D. Jewitt, \& K. Keil (Tucson, AZ: Univ. Arizona Press), 573

Nakajima, T., Kulkarni, S. R., Gorham, P. W., et al. 1989, AJ, 97, 1510

Parker, R. J., \& Quanz, S. P. 2012, MNRAS, 419, 2448

Quanz, S. P., Crepp, J. R., Janson, M., et al. 2012, ApJ, 754, 127

Quanz, S., Meyer, M., Kenworthy, M., et al. 2010, ApJ, 722, 49

Quillen, A. C. 2006, MNRAS, 372, L14

Riaud, P., Mawet, D., \& Magette, A. 2012, A\&A, 545, A150

Rousset, G., Lacombe, F., Puget, P., et al. 2003, Proc. SPIE, 4839, 140

Skemer, A. J., Hinz, P. M., Esposito, S., et al. 2012, ApJ, 753, 14

Spiegel, D. S., \& Burrows, A. 2012, ApJ, 745, 174

Tuthill, P., Lloyd, J., Ireland, M., et al. 2006, Proc. SPIE, 6272, 62723A

Tuthill, P. G., Monnier, J. D., Danchi, W. C., Wishnow, E. H., \& Haniff, C. A. 2000, PASP, 112, 555

van der Bliek, N. S., Manfroid, J., \& Bouchet, P. 1996, A\&AS, 119, 547

Vigan, A., Patience, J., Marois, C., et al. 2012, A\&A, 544, A9

Wildi, F. P., Brusa, G., Riccardi, A., et al. 2002, Proc. SPIE, 4494, 11

Wilson, J. C., Hinz, P. M., Skrutskie, M. F., et al. 2008, Proc. SPIE, 7013, 70133A

Wyatt, M. C., Smith, R., Su, K. Y. L., et al. 2007, ApJ, 663, 365 